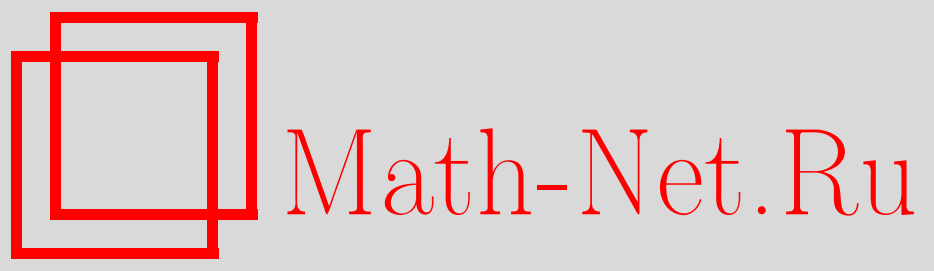

Н. Эйлер, П. Г. Л. Лич, Аспекты собственных дифференциальных последовательностей обыкновенных дифференциальных уравнений, ТMФ, 2009, том 159, номер 1, 64-80

DOI: https://doi.org/10.4213/tmf6333

Использование Общероссийского математического портала Math-Net.Ru подразумевает, что вы прочитали и согласны с пользовательским соглашением http://www . mathnet.ru/rus/agreement

Параметры загрузки:

IP : 3.91 .87 .62

26 апреля 2023 г., 16:14:10

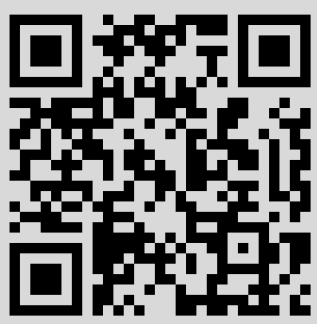




\title{
АСПЕКТЫ СОБСТВЕННЫХ ДИФФЕРЕНЦИАЛЬНЫХ ПОСЛЕДОВАТЕЛЬНОСТЕЙ ОБЫКНОВЕННЫХ ДИФФЕРЕНЦИАЛЬНЫХ УРАВНЕНИЙ
}

\begin{abstract}
Определена собственная дифференциальная последовательность обыкновенных дифференциальных уравнений и для нее предложен метод вывода дополнительной последовательности интегралов. Описаны некоторые общие свойства этих последовательностей, которые иллюстрируются несколькими примерами.
\end{abstract}

Ключевые слова: последовательность обыкновенных дифференциальных уравнений, оператор рекурсии, симметрийный анализ обыкновенных дифференциальных уравнений, интегрируемые обыкновенные дифференциальные уравнения.

\section{1. ВВЕДЕНИЕ}

В недавней работе [1] был проведен симметрийный анализ и анализ Пенлеве двух последовательностей обыкновенных дифференциальных уравнений, а именно последовательности Риккати и последовательности Ермакова-Пинни. Авторы работы [2] использовали анализ сингулярностей и явное решение последовательности Риккати для того, чтобы продемонстрировать некоторые конкретные результаты, которые могут возникать при анализе сингулярностей. Вслед за этим в работе [3] были подробно исследованы свойства симметрии и сингулярности последовательности Риккати.

Целью данной работы является определение собственной дифференциальной последовательности обыкновенных дифференциальных уравнений и обсуждение ее интегрируемости. Мы также вводим дополнительную последовательность, для высших членов которой порядок уравнений не увеличивается - он задается первым уравнением. Этот подход может оказаться полезным при интегрировании и в ряде случаев может приводить к прямому способу получения первых интегралов уравнений дифференциальной последовательности.

${ }^{*}$ Department of Mathematics, Luleå University of Technology, Luleå, Sweden.

E-mail: Norbert.Euler@sm.luth.se

${ }^{\dagger}$ School of Mathematical Sciences, University of KwaZulu-Natal, Durban, Republic of South Africa. E-mail: leachp@ukzn.ac.za, leach@math.aegean.gr 
Полная классификация всех интегрируемых эволюционных дифференциальных уравнений второго порядка в частных производных дана в работе [4]. Мы, разумеется, не бросаем вызов этому результату, поскольку имеем дело исключительно с рекурсионными операторами для обыкновенных дифференциальных уравнений и, что даже более важно, используем эти рекурсионные операторы для интегрирования последовательности обыкновенных дифференциальных уравнений. Мы не пытаемся дать какую-либо классификацию, а лишь приводим некоторые примеры последовательностей для иллюстрации нашего метода (см. предложение 2 в разделе 3$)$.

Работа построена следующим образом. В разделе 2 даются определения, касающиеся собственных дифференциальных последовательностей, алгебр Ли их симметрий, операторов рекурсии и интегрируемости. В разделе 3 описан метод получения дополнительной последовательности и приводится несколько примеров, иллюстрирующих концепцию совместных и полностью совместных последовательностей, которая дает метод интегрирования. В приложении для некоторых из последовательностей, рассмотренных в разделе 3, приведены подробности их симметрийного анализа на основе точечных групп Ли.

\section{2. ОБЩЕЕ ОПИСАНИЕ}

Рассмотрим переменные $x$ и $u$, где $u=u(x)$ и $u_{x}=d u / d x, u_{x x}=d^{2} u / d x^{2}$; далее обозначения расширяются до $u_{n x}=d^{n} u / d x^{n}$. Рассмотрим дифференциальную последовательность $m$ уравнений

$$
\left\{E_{1}, E_{2}, \ldots, E_{m}\right\}
$$

вида

$$
\begin{aligned}
E_{1} & :=F\left(u, u_{x}, u_{x x}, \ldots, u_{n x}\right)=0 \\
E_{2} & :=R^{[k]}[u] F\left(u, u_{x}, u_{x x}, \ldots, u_{n x}\right)=0, \\
E_{3} & :=\left(R^{[k]}[u]\right)^{2} F\left(u, u_{x}, u_{x x}, \ldots, u_{n x}\right)=0, \\
\left.\ldots \ldots \ldots \ldots \ldots \ldots \ldots \ldots \ldots, \ldots \ldots \ldots, \ldots \ldots, u_{n x}\right)=0, &
\end{aligned}
$$

где $R^{[k]}[u]$ - интегродифференциальный оператор $k$-го порядка,

$$
R^{[k]}[u]=G_{k} D_{x}^{k}+G_{k-1} D_{x}^{k-1}+\cdots+G_{0}+Q D_{x}^{-1} \circ J .
$$

Оператор, сопряженный оператору $R^{[k]}[u]$, имеет вид

$$
\left(R^{[k]}\right)^{*}[u]=\sum_{i=0}^{k}(-1)^{i} D_{x}^{i} \circ G_{i}-J D_{x}^{-1} \circ Q .
$$

Будем говорить, что $E_{1}$ - затравочное уравнение дифференциальной последовательности (2.2). Заметим, что в (2.2) второе уравнение имеет порядок $n+k$, третье уравнение - порядок $n+2 k$, а $m$-е уравнение - порядок $n+(m-1) k$.

3 Теоретическая и математическая физика, т. 159, № 1, 2009 г. 
Пусть $L_{E_{i}}$ обозначает линейный оператор, действующий следующим образом:

$$
L_{E_{i}}[u]=\frac{\partial E_{i}}{\partial u}+\frac{\partial E_{i}}{\partial u_{x}} D_{x}+\frac{\partial E_{i}}{\partial u_{2 x}} D_{x}^{2}+\cdots+\frac{\partial E_{i}}{\partial u_{q x}} D_{x}^{q},
$$

a $L_{E_{i}}^{*}-$ оператор, сопряженный $L_{E_{i}}[u]$ :

$$
L_{E_{i}}^{*}[u]=\frac{\partial E_{i}}{\partial u}-D_{x} \circ \frac{\partial E_{i}}{\partial u_{x}}+D_{x}^{2} \circ \frac{\partial E_{i}}{\partial u_{2 x}}+\cdots+(-1)^{q} D_{x}^{q} \circ \frac{\partial E_{i}}{\partial u_{q x}} .
$$

Пусть $Z^{i}\left(E_{i}\right)$ обозначает генератор вертикальной симметрии уравнения $E_{i}$ из последовательности (2.2),

$$
Z^{i}\left(E_{i}\right)=Q\left(x, u, u_{x}, u_{x x}, u_{3 x}, \ldots, u_{j x}\right) \partial_{u},
$$

где необходимое и достаточное условие инвариантности уравнения $E_{i}$ имеет вид $\left.L_{E_{i}} Q\right|_{E_{i}=0}=0$. Заметим, что выражение (2.7) включает генераторы точечных симметрий $\Gamma_{i}=\xi(x, t, u) \partial_{x}+\eta(x, t, u) \partial_{u}$ с характеристикой симметрии $Q\left(x, u, u_{x}\right)=$ $\xi(x, u) u_{x}-\eta(x, u)$ и эквивалентной вертикальной формой $Z^{i}=\left[\xi(x, u) u_{x}-\eta(x, u)\right] \partial_{u}$.

ОПРЕДЕЛЕНИЕ 1. Последовательность (2.2) допускает р-мерную алгебру Ли симметрий $\mathcal{L}$, которая линейно порождается линейно независимыми генераторами симметрии $\left\{Z_{1}^{i}\left(E_{i}\right), Z_{2}^{i}\left(E_{i}\right), \ldots, Z_{p}^{i}\left(E_{i}\right)\right\}$, если каждое уравнение в последовательности $\left\{E_{1}, E_{2}, \ldots, E_{m}\right\}(2.2)$ допускает $p$-мерную алгебру Ли симметрий $\mathcal{L}^{\prime}$, изоморфную алгебре $\mathcal{L}$.

ОПРЕДЕЛЕНИЕ 2. Функция $J=J\left(x, u, u_{x}, u_{x x}, \ldots\right)$ является интегрирующим множителем для дифференииальной последовательности (2.2), если $J$ - интегрирующий множитель для каждого уравнения в последовательности.

ОПРЕДЕЛЕНИЕ 3. Оператор $R^{[k]}[u]$ вида (2.3) называется оператором рекурсии $k$-го порядка для дифференциальной последовательности (2.2), если

$$
\begin{aligned}
{\left[L_{E_{i}}[u], R^{[k]}[u]\right] } & =0, & i & =1,2, \ldots, m, \\
\left(R^{[k]}\right)^{*}[u] J_{k} & =\alpha J_{l}, & k, l & =1,2, \ldots, p,
\end{aligned}
$$

где $\alpha$ - ненулевая константа, $p$ - полное число интегрирующих множителей $J_{k}$ для последовательности (2.2) (при некоторых значениях $k$ множители $J_{k}$ могут равняться нулю).

ОПРЕДЕЛЕНИЕ 4. Собственной дифференциалъной последователъностъю обыкновенных дифференциальных уравнений называется дифференциальная последовательность, которая допускает по крайней мере один оператор рекурсии вида (2.3).

ОПРЕДЕЛЕНИЕ 5. Интегрируемая дифференииальная последовательность есть собственная дифференциальная последовательность обыкновенных дифференциальных уравнений, в которой каждое уравнение является интегрируемым. 
ЗАмЕчАниЕ. Под интегрируемым обыкновенным дифференциальным уравнением $n$-го порядка понимается уравнение, допускающее решение $u=\phi\left(x ; c_{1}, \ldots, c_{n}\right)$, где $c_{j}$ - независимые произвольные константы, $j=1,2, \ldots, n$. В менее ограничительном смысле мы требуем, чтобы уравнение $n$-го порядка допускало $n-1$ функционально независимых первых интегралов таких, что общее решение можно выразить в квадратурах. Интегрируемость нелинейного обыкновенного дифференциального уравнения можно также выразить в терминах структуры его сингулярностей в комплексной области. Это известно как свойство Пенлеве: требуется, чтобы решения обладали только сингулярностями в виде подвижных полюсов (см., например, недавний обзор [5], посвященный свойству Пенлеве). Исследование свойства Пенлеве собственных дифференциальных последовательностей может представлять интерес, но эта тема находится за рамками настоящей статьи. Сошлемся на работы [2], [3], где анализ особенностей использован для изучения интегрируемости последовательности Риккати.

Пусть

$$
E_{i}:=u_{q x}-f_{i}\left(x, u, u_{x}, u_{x x}, \ldots, u_{(q-1) x}\right)=0, \quad i=1,2, \ldots, m,
$$

где $q=n+(i-1) k$. Введем следующий оператор полной производной:

$$
D_{E_{i}}=\left.D_{x}\right|_{E_{i}=0}=\frac{\partial}{\partial x}+\sum_{j=0}^{q-1} u_{j x} \frac{\partial}{\partial u_{(j-1) x}}+f_{i}\left(x, u, u_{x}, \ldots, u_{(q-1) x}\right) \frac{\partial}{\partial u_{(q-1) x}} .
$$

ПрЕДЛОЖЕНИЕ 1. Функиия $J_{s}$ является интегрирующим множителем для последовательности (2.9), если и только если выполнены условия

$$
\begin{gathered}
\left.L_{E_{i}[u]}^{*} J_{s}\left(x, u, u_{x}, \ldots\right)\right|_{E_{i}=0}=0, \quad i=1,2, \ldots, m, \\
\frac{\partial J_{s}}{\partial u_{(q-2 r) x}}+\sum_{j=1}^{2 r-1}(-1)^{j-1} \frac{\partial}{\partial u_{(q-1) x}}\left\{D_{E_{i}}^{j-1}\left(\frac{\partial f_{i}}{\partial u_{(j+q-2 r) x}} J_{s}\right)\right\}+ \\
+\frac{\partial}{\partial u_{(q-1) x}}\left(D_{E_{i}}^{2 r-1} J_{s}\right)=0, \\
s=1,2, \ldots, p, \quad r=1,2, \ldots,\left[\frac{q}{2}\right], \quad i=1,2, \ldots, m .
\end{gathered}
$$

Здесь $[q / 2]$ - наибольшее натуральное число, не превосходящее числа q/2, а р полное число интегрирующих множителей $J_{s}$ для последовательности (2.9).

ЗАмЕчАНИЕ. ВЫвод необходимых и достаточных условий для интегрирующих множителей одного обыкновенного дифференциального уравнения $n$-го порядка приведен в книге [6]. Предложение 1 является естественным обобщением этого результата на собственные дифференциальные последовательности обыкновенных 
дифференциальных уравнений. Заметим, что из условия (2.11a) следует, что каждый множитель $J_{s}$ является сопряженной симметрией для каждого уравнения в последовательности, а из условия (2.11б) следует, что эта сопряженная симметрия является интегрирующим множителем для каждого члена последовательности.

ПримеР 1. Рассмотрим затравочное уравнение $u_{x x}+u_{x}^{2}=0$ с дифференциальным оператором $R[u]=D_{x}+u_{x}$. Тем самым задана собственная дифференциальная последовательность

$$
R^{j}[u]\left(u_{x x}+u_{x}^{2}\right)=0, \quad j=1,2, \ldots, m,
$$

с интегрирующими множителями нулевого порядка $J_{1}(x, u)=e^{u}, J_{2}(x, u)=x e^{u}$. Здесь $R^{*}[u] e^{u}=0, R^{*}[u]\left(x e^{u}\right)=-e^{u}$.

В следующем разделе будет показано, что последовательность (2.12) является интегрируемой последовательностью обыкновенных дифференциальных уравнений; мы также рассмотрим ее свойства.

\section{3. ДОПОЛНИТЕЛЬНОЕ ОПИСАНИЕ}

Для последовательности $\left\{E_{1}, E_{2}, \ldots, E_{m}\right\}(2.2)$ интересно построить дополнительную последовательность

$$
\left\{\widetilde{E}_{1}, \widetilde{E}_{2}, \ldots, \widetilde{E}_{m}\right\}
$$

в которой порядок дифференциальных уравнений не возрастает, а фиксирован затравочным уравнением $\widetilde{E}_{1}$. Тогда для одного и того же затравочного уравнения $E_{1}=\widetilde{E}_{1}$ две последовательности (2.1) и (3.1) должны быть совместны или полностью совместны. Дадим определение этих понятий.

ОПРЕДЕлЕНиЕ 6 . Два уравнения $E_{j}$ и $\widetilde{E}_{j}$ из соответствующих последовательностей (2.2) и (3.1) называются совместными, если эти уравнения допускают по крайней мере одно совместное решение. Два уравнения называются полностъю совместными, если общее решение уравнения $\widetilde{E}_{j}$ является общим решением уравнения $E_{j}$. Две последовательности $\left\{E_{1}, E_{2}, \ldots, E_{m}\right\}$ и $\left\{\widetilde{E}_{1}, \widetilde{E}_{2}, \ldots, \widetilde{E}_{m}\right\}$ из $m$ уравнений с одним и тем же затравочным уравнением $E_{1}=\widetilde{E}_{1}$ называются совместными, если каждое уравнение последовательности допускает по крайней мере одно совместное решение для соответствующих членов в обеих последовательностях. Последовательности называются полностъю совместными, если общее решение уравнения $\widetilde{E}_{j}$ дает общее решение уравнения $E_{j}$ для всех $j=1,2, \ldots, m$. Последовательность (3.1) называется дополнительной для (2.2), если эти две последовательности являются как минимум совместными.

Поскольку порядок уравнений в дополнительной последовательности (3.1) не возрастает, уравнения, составляющие дополнительную последовательность, должны определять интегралы уравнений собственной дифференциальной последовательности (2.1) для того, чтобы обеспечить совместность ее решений. Имеем следующий факт. 
ПреДЛОЖЕНИЕ 2. Рассмотрим собственную дифференииальную последовательность $\left\{E_{1}, E_{2}, \ldots, E_{m}\right\}$ с оператором рекурсии $R^{[k]}[u]$. Дополнительная последовательность $\left\{\widetilde{E}_{1}, \widetilde{E}_{2}, \ldots, \widetilde{E}_{m}\right\}$ вида

$$
\begin{aligned}
\widetilde{E}_{1} & :=F\left(u, u_{x} u_{x x}, \ldots, u_{n x}\right)=0, \\
\widetilde{E}_{j+1} & :=F\left(u, u_{x} u_{x x}, \ldots, u_{n x}\right)= \\
& =Q_{j}\left(x, u, u_{x}, \ldots, \omega^{1}, \omega^{2}, \ldots, \omega^{\ell} ; c_{1}, c_{2}, \ldots, c_{s}\right), \quad j=1,2, \ldots, m-1,
\end{aligned}
$$

совместна с собственной дифференииальной последовательностью $\left\{E_{1}, E_{2}, \ldots\right.$ $\left.\ldots, E_{m}\right\}, E_{1}=\widetilde{E}_{1}$, если

$$
\begin{aligned}
& R^{[k]} Q_{1}=0, \\
& R^{[k]} Q_{i}=Q_{i-1}, \quad i=2,3, \ldots, m .
\end{aligned}
$$

Здесь $\omega^{1}, \omega^{2}, \ldots, \omega^{\ell}-$ нелокальные координаты, определяемые формулами

$$
\begin{aligned}
& \frac{d \omega^{1}}{d x}=g_{1}(u) \\
& \frac{d \omega^{2}}{d x}=g_{2}\left(\omega^{1}\right), \quad \frac{d \omega^{3}}{d x}=g_{3}\left(\omega^{2}\right), \quad \ldots, \quad \frac{d \omega^{\ell}}{d x}=g_{\ell}\left(\omega^{\ell-1}\right)
\end{aligned}
$$

с некоторыми дифференцируемыми функииями $g_{k}$.

Ниже мы рассмотрим несколько примеров собственных дифференциальных последовательностей, совместных или полностью совместных со своими дополнительными последовательностями. Примеры достаточно просты для иллюстрации метода построения и исследования свойств соответствующих последовательностей. Попытки классификации в настоящей работе не предпринимаются, но будут рассмотрены в последующих работах. Для наших примеров мы берем четыре затравочных уравнения, которые являются частью списка линеаризуемых эволюционных уравнений второго порядка в размерности $(1+1)$ из работы [7]:

$$
\begin{gathered}
u_{x x}+u_{x}^{2}=0, \quad u_{x x}+h(u) u_{x}^{2}=0, \\
u_{x x}+\lambda u_{x}-\frac{h^{\prime}(u)}{h(u)} u_{x}^{2}+h(u)=0, \quad u_{x x}+u u_{x}=0
\end{gathered}
$$

с произвольными дифференцируемыми функциями $h$; здесь штрих обозначает производную по $u$.

ПримеР 2. Сначала рассмотрим собственную дифференциальную последовательность (2.12), введенную в примере 1 , где $R[u]=D_{x}+u_{x}$. В этом случае собственная дифференциальная последовательность есть

$$
\begin{aligned}
& E_{1}:=F\left(u, u_{x} u_{x x}\right)=u_{x x}+u_{x}^{2}=0, \\
& E_{2}:=R[u] F\left(u, u_{x} u_{x x}\right)=u_{3 x}+3 u_{x} u_{x x}+u_{x}^{3}=0, \\
& E_{3}:=R^{2}[u] F\left(u, u_{x} u_{x x}\right)=u_{4 x}+4 u_{x} u_{3 x}+3 u_{x x}^{2}+6 u_{x}^{2} u_{x x}+u_{x}^{4}=0 \text {, } \\
& E_{m}:=R^{m-1}[u] F\left(u, u_{x} u_{x x}\right)=u_{(m+1) x}+\cdots=0 .
\end{aligned}
$$


Применим предложение 2 для вычисления дополнительной последовательности с затравочным уравнением (3.5a). Второй член в дополнительной последовательности имеет вид $u_{x x}+u_{x}^{2}=Q_{1}\left(x, u, u_{x}, \ldots\right)$ при условии $R[u] Q_{1}\left(x, u, u_{x}, \ldots\right)=0$. Данное условие записывается как $D_{x}\left(Q_{1}\right)=-u_{x} Q_{1}$ и имеет общее решение

$$
Q_{1}\left(u, c_{1}\right)=c_{1} e^{-u}
$$

где $c_{1}-$ произвольная постоянная интегрирования. Таким образом, второй член в дополнительной последовательности есть $u_{x x}+u_{x}^{2}=c_{1} e^{-u}$. В дополнительной последовательности третий член, отвечающий (3.5в), принимает вид $u_{x x}+u_{x}^{2}=$ $Q_{2}\left(x, u, u_{x}, \ldots\right)$ при условии $R[u] Q_{2}\left(x, u ; c_{1}, c_{2}\right)=Q_{1}\left(u ; c_{1}\right)$, которое допускает общее решение $Q_{2}\left(x, u ; c_{1}, c_{2}\right)=c_{1} x e^{-u}+c_{2} e^{-u}$, где $c_{2}$ - еще одна постоянная интегрирования. Таким образом, третий член в дополнительной последовательности имеет вид $u_{x x}+u_{x}^{2}=e^{-u}\left(c_{1} x+c_{2}\right)$, что можно переписать как $u_{x x}+u_{x}^{2}=e^{-u} D_{x}^{-1} c_{1}$. Следующий член в дополнительной последовательности есть

$$
u_{x x}+u_{x}^{2}=e^{-u}\left(\frac{1}{2} c_{1} x^{2}+c_{2} x+c_{3}\right) \equiv e^{-u} D_{x}^{-2} c_{1} .
$$

Функции $Q_{k}$ в $E_{k+1}$ таковы:

$$
Q_{k}=e^{-u} q(x) \quad \text { при } \quad q^{(k)}(x)=0 \quad \Leftrightarrow \quad q(x)=\sum_{j=1}^{k} \frac{c_{j}}{(k-j) !} x^{k-j} .
$$

Таким образом, мы заключаем, что последовательность, дополнительная к дифференциальной последовательности (3.5), имеет вид

$$
\begin{aligned}
& \widetilde{E}_{1}:=u_{x x}+u_{x}^{2}=0, \\
& \widetilde{E}_{j}:=u_{x x}+u_{x}^{2}=e^{-u} D_{x}^{-(j-2)} c_{1}, \quad j=2,3, \ldots, m,
\end{aligned}
$$

где $D_{x}^{-q}$ - это $q \in \mathbb{N}$ композиций интегрального оператора $D_{x}^{-1}$ (при $D_{x}^{0}:=1$ ). Явным образом дополнительная последовательность записывается как

$$
\begin{aligned}
& \widetilde{E}_{1}:=u_{x x}+u_{x}^{2}=0, \\
& \widetilde{E}_{2}:=u_{x x}+u_{x}^{2}=Q_{1}, \quad \text { где } \quad Q_{1}=e^{-u} c_{1}, \\
& \widetilde{E}_{3}:=u_{x x}+u_{x}^{2}=Q_{2}, \quad \text { где } \quad Q_{2}=e^{-u}\left(c_{1} x+c_{2}\right), \\
& \widetilde{E}_{4}:=u_{x x}+u_{x}^{2}=Q_{3}, \quad \text { где } \quad Q_{3}=e^{-u}\left(\frac{1}{2} c_{1} x^{2}+c_{2} x+c_{3}\right), \\
& \ldots \ldots \ldots \ldots \ldots \ldots \ldots \ldots \ldots \ldots \ldots \ldots \ldots \ldots \ldots \ldots \ldots \ldots \ldots \ldots \ldots \ldots \ldots \\
& \widetilde{E}_{m}:=u_{x x}+u_{x}^{2}=Q_{m-1}, \quad \text { где } \quad Q_{m-1}=e^{-u}\left(\sum_{j=1}^{m-1} \frac{c_{j}}{(m-j-1) !} x^{m-j-1}\right) .
\end{aligned}
$$


Легко установить, что собственная дифференциальная последовательность (3.5) является интегрируемой дифференциальной последовательностью, поскольку каждый ее член может быть линеаризован заменой переменных $w(X)=u_{x} e^{u}, X=x$. Более того, дополнительная последовательность (3.7) линеаризуется с помощью замены переменных $w(X)=e^{u}, X=x$. Чтобы установить совместность или полную совместность двух последовательностей (3.5) и (3.7), рассмотрим внимательнее соответствующие члены.

Сравним члены $E_{2}$ и $\widetilde{E}_{2}$ : первый интеграл для $E_{2}$ имеет вид $\widetilde{E}_{2}$, а именно $c_{1}=$ $e^{u}\left(u_{x x}+u_{x}^{2}\right)$. Поэтому общее решение уравнения $\widetilde{E}_{2}$ дает общее решение для $E_{2}$, где $c_{1}$ - одна из постоянных интегрирования для $E_{2}$. Итак, два уравнения $E_{2}$ и $\widetilde{E}_{2}$ полностью совместны.

Сравним члены $E_{3}$ и $\widetilde{E}_{3}$ : второй интеграл для $E_{3}$ имеет вид $\widetilde{E}_{3}$, а именно $c_{1} x+c_{2}=$ $e^{u}\left(u_{x x}+u_{x}^{2}\right)$. Поэтому общее решение уравнения $\widetilde{E}_{3}$ дает общее решение для $E_{3}$ (где $c_{1}$ и $c_{2}$ - две постоянные интегрирования для $E_{3}$ ), и два уравнения $E_{3}$ и $\widetilde{E}_{3}$ полностью совместны. Подобный аргумент примени́м и ко всем остальным уравнениям из собственной дифференциальной последовательности (3.5). В результате мы заключаем, что последовательности (3.5) и (3.7) являются полностью совместными.

Другое интересное свойство последовательностей (3.5) и (3.7) состоит в том, что симметрийные характеристики $\eta_{j}$ симметрий $\Gamma_{j}^{s}=\eta_{j}(x, u) \partial_{u}$ решения уравнений (3.5) записываются через функции $Q_{1}, Q_{2}, \ldots$ из дополнительной последовательности (3.7). В частности, симметрийная характеристика $\eta_{j}$ для симметрий решения уравнения $E_{j}$ из $(3.5)$ выражается как функция $Q_{j+1}$ из уравнения $\widetilde{E}_{j+2}$ в $(3.7)$ для всех $j=1,2, \ldots, m$. Например, уравнение $E_{1}:=u_{x x}+u_{x}^{2}=0$ допускает симметрию решения $\Gamma_{1}^{s}=Q_{2} \partial_{u}$, где $Q_{2}=e^{-u}\left(c_{1} x+c_{2}\right)$ соответствует второму выражению в (3.7в).

Заметим, что полный набор всех точечных симметрий для собственной дифференциальной последовательности (3.5) таков:

для $E_{1}$ полный набор точечных симметрий Ли есть

$$
\left\{e^{-u} \partial_{u}, x e^{-u} \partial_{u}, \partial_{u}, \partial_{x}, x \partial_{+} \frac{1}{2} \partial_{u}, x^{2} \partial_{x}+x \partial_{u}, e^{u} \partial_{x}, x e^{u} \partial_{x}+e^{u} \partial_{u}\right\}
$$

для $E_{k}, k=2,3, \ldots, m$, полный набор точечных симметрий Ли есть

$$
\left\{q(x) e^{-u} \partial_{u}, \partial_{u}, \partial_{x}, x \partial_{x}+\frac{1}{2}(n-1) \partial_{u}, x^{2} \partial_{x}+(n-1) x \partial_{u}\right\},
$$

где $n$ - порядок дифференциального уравнения $E_{k}$ и $q^{(n)}(x)=0$.

Свойства точечных симметрий Ли дополнительной последовательности (3.7) рассматриваются в приложении. 
ПримеР 3. Рассмотрим затравочное уравнение $u_{x x}+h(u) u_{x}^{2}=0$ с рекурсионным оператором $R[u]=D_{x}+h(u) u_{x}$. Тем самым определена собственная дифференциальная последовательность вида

$$
\begin{aligned}
E_{1} & :=u_{x x}+h(u) u_{x}^{2}=0, \\
E_{j+1} & :=R^{j}[u]\left(u_{x x}+h(u) u_{x}^{2}\right)=0, \quad j=1,2, \ldots, m-1 .
\end{aligned}
$$

Применим предложение 2 для вычисления функций $Q_{j}$ тем же способом, как и в примере 2. Это приводит к дополнительной последовательности

$$
\begin{gathered}
\widetilde{E}_{1}:=u_{x x}+h(u) u_{x}^{2}=0 \\
\widetilde{E}_{j+1}:=u_{x x}+h(u) u_{x}^{2}=\exp \left[-\int h(u) d u\right] D_{x}^{-(j-1)} c_{1}, \\
j=1,2, \ldots, m-1 .
\end{gathered}
$$

Легко показать, что последовательность (3.10) и ее дополнительная последовательность (3.11) полностью совместны и что (3.10) - интегрируемая последовательность. Линеаризация и точечные симметрии Ли последовательности (3.11) рассмотрены в приложении.

ПРимеР 4. Рассмотрим затравочное уравнение

$$
u_{x x}+\lambda u_{x}-\frac{h^{\prime}(u)}{h(u)} u_{x}^{2}+h(u)=0
$$

с рекурсионным оператором $R[u]=D_{x}-\frac{h^{\prime}(u)}{h(u)} u_{x}$. Тем самым определены собственная дифференциальная последовательность

$$
\begin{aligned}
E_{1} & :=u_{x x}+\lambda u_{x}-\frac{h^{\prime}(u)}{h(u)} u_{x}^{2}+h(u)=0, \\
E_{j+1} & :=R^{j}[u]\left(u_{x x}+\lambda u_{x}-\frac{h^{\prime}(u)}{h(u)} u_{x}^{2}+h(u)\right)=0, \quad j=1,2, \ldots, m-1,
\end{aligned}
$$

и ее дополнительная последовательность

$$
\begin{gathered}
\widetilde{E}_{1}:=u_{x x}+\lambda u_{x}-\frac{h^{\prime}(u)}{h(u)} u_{x}^{2}+h(u)=0, \\
\widetilde{E}_{j+1}:=u_{x x}+\lambda u_{x}-\frac{h^{\prime}(u)}{h(u)} u_{x}^{2}+h(u)=h(u) D_{x}^{(j-1)} c_{1}, \\
j=1,2, \ldots, m-1,
\end{gathered}
$$

где $h$ - произвольная дифференцируемая функция. Как и последовательности из примеров 2 и 3, последовательности (3.12) и (3.13) полностью совместны, а (3.12) интегрируемая последовательность. Подробности приведены в приложении. 
Пример 5. С уравнением Бюргерса $u_{x x}+u u_{x}=u_{t}$ можно (с помощью стандартной симметрийной редукции в соответствии с $t$-трансляционной инвариантностью) связать уравнение

$$
u_{x x}+u u_{x}=0
$$

у которого тот же интегродифференциальный рекурсионный оператор

$$
R[u]=D_{x}+\frac{1}{2} u+\frac{1}{2} u_{x} D_{x}^{-1} \circ 1 .
$$

Собственная дифференциальная последовательность, которую мы назовем последовательностъю Бюргерса, имеет вид

$$
\begin{aligned}
E_{1} & :=u_{x x}+u u_{x}=0, \\
E_{j+1} & :=R^{j}[u]\left(u_{x x}+u u_{x}\right)=0, \quad j=1,2, \ldots, m .
\end{aligned}
$$

Теперь построим дополнительную последовательность Бюргерса, следуя предложению 2. Решение уравнения $R[u] Q_{1}=0$ есть

$$
Q_{1}=\left(-2 A \exp \left[-\frac{1}{2} \int u d x\right]+2 B \exp \left[-\frac{1}{2} \int u d x\right] \int \exp \left[\frac{1}{2} \int u d x\right] d x\right)_{x}
$$

где $A$ и $B$ - постоянные интегрирования. Это уравнение является интегродифференциальным. Его можно свести к обыкновенному дифференциальному уравнению, положив

$$
w=\int \exp \left[\frac{1}{2} \int u d x\right] d x
$$

Тогда уравнение

$$
\begin{aligned}
u_{x x}+u u_{x}= & \left(-2 A \exp \left[-\frac{1}{2} \int u d x\right]+\right. \\
& \left.+2 B \exp \left[-\frac{1}{2} \int u d x\right] \int \exp \left[\frac{1}{2} \int u d x\right] d x\right)_{x}
\end{aligned}
$$

принимает вид

$$
\frac{w_{4 x}}{w_{x}}-\frac{w_{x x} w_{3 x}}{w_{x}^{2}}=\frac{A w_{x x}}{w_{x}^{2}}+B\left(1-\frac{w w_{x x}}{w_{x}^{2}}\right)
$$

Подобным образом получаем, что уравнение $R[u] Q_{2}=Q_{1}$ имеет решение

$$
\begin{aligned}
Q_{2}= & \left\{2 C \exp \left[-\frac{1}{2} \int u d x\right] \int \exp \left[\frac{1}{2} \int u d x\right] d x-2 A x \exp \left[-\frac{1}{2} \int u d x\right]+\right. \\
& \left.+2 B \exp \left[-\frac{1}{2} \int u d x\right] \int\left(\int \exp \left[\frac{1}{2} \int u d x\right] d x\right) d x\right\}_{x},
\end{aligned}
$$


где $C$ - также постоянная интегрирования, а интегродифференциальное уравнение принимает вид

$$
\begin{aligned}
u_{x x}+u u_{x}= & \left\{2 C \exp \left[-\frac{1}{2} \int u d x\right] \int \exp \left[\frac{1}{2} \int u d x\right] d x-2 A x \exp \left[-\frac{1}{2} \int u d x\right]+\right. \\
& \left.+2 B \exp \left[-\frac{1}{2} \int u d x\right] \int\left(\int \exp \left[\frac{1}{2} \int u d x\right] d x\right) d x\right\}_{x} .
\end{aligned}
$$

Соответствующее обыкновенное дифференциальное уравнение высшего порядка есть

$$
\frac{w_{5 x}}{w_{x x}}-\frac{w_{3 x} w_{4 x}}{w_{x x}^{2}}=C\left(1-\frac{w_{x} w_{3 x}}{w_{x x}^{2}}\right)+A\left(\frac{x w_{3 x}}{w_{x x}^{2}}-\frac{1}{w_{x x}}\right)+B\left(\frac{w_{x}}{w_{x x}}-\frac{w w_{3 x}}{w_{x x}^{2}}\right),
$$

где теперь

$$
w=\int\left(\int \exp \left[\frac{1}{2} \int u d x\right] d x\right) d x
$$

или, эквивалентно,

$$
u=2 \frac{w_{3 x}}{w_{x x}} .
$$

Можно, очевидно, продолжить в том же духе. В отличие от примеров 2-4, в которых рекурсионный оператор не содержал обратной производной, для получения обыкновенного дифференциального уравнения следует переопределить зависимые переменные, как это сделано в (3.18) и (3.23) (с очевидным обобщением на высшие элементы последовательности). Заметим, что члены в левых частях уравнений (3.20) и (3.22) имеют один и тот же вид, за исключением увеличивающегося порядка каждой производной. Следовательно, если мы хотим иметь последовательность дифференциальных уравнений из $m$ элементов, основанную на дифференциальном уравнении (3.14) и его операторе рекурсии (3.15), то все дифференциальные уравнения, составляющие последовательность, следует записать в терминах дифференциального уравнения порядка $m+2$. Альтернативой является интегродифференциальное уравнение с возрастающей нелокальностью.

Таким образом, мы приходим к заключению, что три первых члена в дополнительной последовательности принимают вид

$$
\begin{aligned}
\widetilde{E}_{1}(w) & :=\frac{w_{5 x}}{w_{x x}}-\frac{w_{3 x} w_{4 x}}{w_{x x}^{2}}=0 \Leftrightarrow \\
& \Leftrightarrow\left(\frac{w_{4 x}}{w_{x x}}\right)_{x}=0 \Leftrightarrow w_{4 x}=k_{1} w_{2 x} \\
\widetilde{E}_{2}(w) & :=\frac{w_{5 x}}{w_{x x}}-\frac{w_{3 x} w_{4 x}}{w_{x x}^{2}}=\frac{A w_{3 x}}{w_{x x}^{2}}+B\left(1-\frac{w_{x} w_{3 x}}{w_{x x}^{2}}\right) \Leftrightarrow \\
& \Leftrightarrow\left(\frac{w_{4 x}}{w_{x x}}\right)_{x}=-\left(\frac{A}{w_{x x}}\right)_{x}+B\left(\frac{w_{x}}{w_{x x}}\right)_{x} \Leftrightarrow \\
& \Leftrightarrow w_{4 x}=a_{1} w_{x x}+B w_{x}-A,
\end{aligned}
$$




$$
\begin{aligned}
\widetilde{E}_{3}(w):= & \frac{w_{5 x}}{w_{x x}}-\frac{w_{3 x} w_{4 x}}{w_{x x}^{2}}=C\left(1-\frac{w_{x} w_{3 x}}{w_{x x}^{2}}\right)+A\left(\frac{x w_{3 x}}{w_{x x}^{2}}-\frac{1}{w_{x x}}\right)+ \\
& +B\left(\frac{w_{x}}{w_{x x}}-\frac{w w_{3 x}}{w_{x x}^{2}}\right) \Leftrightarrow \\
\Leftrightarrow & \left(\frac{w_{4 x}}{w_{x x}}\right)_{x}=C\left(\frac{w_{x}}{w_{x x}}\right)_{x}-A\left(\frac{x}{w_{x x}}\right)_{x}+B\left(\frac{w}{w_{x x}}\right)_{x} \Leftrightarrow \\
\Leftrightarrow & w_{4 x}=a_{2} w_{x x}+C w_{x}+B w-A x .
\end{aligned}
$$

Однако такую дифференциальную последовательность не следует путать с дифференциальной последовательностью нормального типа, поскольку если значение $m$ увеличивается, то как левая, так и правая части уравнений, а также оператор рекурсии должны быть переопределены.

Для установления совместности первых трех членов двух последовательностей следует записать последовательности (3.16) и (3.26) в терминах одной и той же переменной $w$, т.е. необходимо применить преобразование (3.25) и преобразовать первые три члена дифференциальной последовательности (3.16) так, чтобы записать их в терминах переменной $w$. Получаем

$$
\begin{aligned}
& E_{1}(w):=\frac{w_{5 x}}{w_{x x}}-\frac{w_{3 x} w_{4 x}}{w_{x x}^{2}}=0 \quad \Leftrightarrow \quad\left(\frac{w_{4 x}}{w_{x x}}\right)_{x}=0 \quad \Leftrightarrow \quad w_{3 x}=k_{1} w_{x}+k_{11}, \\
& E_{2}(w):=\frac{w_{6 x}}{w_{x x}}-\frac{w_{3 x} w_{5 x}}{w_{x x}^{2}}=0 \quad \Leftrightarrow \quad\left(\frac{w_{5 x}}{w_{x x}}\right)_{x}=0 \quad \Leftrightarrow \quad w_{4 x}=k_{2} w_{x}+k_{21}, \\
& E_{3}(w):=\frac{w_{7 x}}{w_{x x}}-\frac{w_{3 x} w_{6 x}}{w_{x x}^{2}}=0 \quad \Leftrightarrow \quad\left(\frac{w_{6 x}}{w_{x x}}\right)_{x}=0 \quad \Leftrightarrow \quad w_{5 x}=k_{3} w_{x}+k_{31},
\end{aligned}
$$

где все $k_{j}$ и $k_{i j}$ - постоянные интегрирования.

Сравним последнее уравнение в (3.26б) с (3.27б), а последнее уравнение в (3.26в) с (3.27в), получим, что эти две последовательности совместны, но не полностью совместны. В частности, уравнение $w_{4 x}=B w_{x}-A$, которое представляет собой последнее уравнение (3.26б) при $a_{1}=0$, является вторым интегралом для уравнения $E_{2}$, а именно для (3.27б). Однако тот же самый второй интеграл для (3.27в) следует из последнего уравнения в $(3.26 \mathrm{~B})$, где два дополнительных параметра $a_{2}$ и $C$ должны для совместности равняться нулю. Поэтому высшие члены дополнительной последовательности не дают дополнительных параметров для интегрирования дифференциальной последовательности (3.16), и мы заключаем, что две данные последовательности всего лишь обладают совместными специальными решениями. Однако собственная дифференциальная последовательность (3.16) интегрируема, поскольку каждый ее член можно линеаризовать. То же, разумеется, верно для дополнительной последовательности (3.26).

В отличие от примеров 2-4, в данном случае нет сохранения точечных симметрий Ли в дополнительной последовательности. Для уравнений (3.26а), (3.26б), (3.26в) соответственно получаем

$$
\Gamma_{1}=\partial_{x}, \quad \Gamma_{2}=\partial_{w}, \quad \Gamma_{3}=x \partial_{x}, \quad \Gamma_{4}=x \partial_{w}, \quad \Gamma_{5}=w \partial_{w},
$$




$$
\begin{gathered}
\Gamma_{1}=\partial_{x}, \quad \Gamma_{2}=\partial_{w}, \quad \Gamma_{6}=(A x-B w) \partial_{w}, \\
\Gamma_{7}=B \partial_{x}+A \partial_{w}, \quad \Gamma_{8}=\left(A B x-B^{2} w-A C\right) \partial_{w} .
\end{gathered}
$$

Выше мы рассматривали только три первых члена последовательности. Закончим этот пример следующими утверждениями о полной последовательности (3.16).

ПреДЛОЖЕНИЕ 3. Для дополнителъной дифференииалъной последователъности Бюргерса, записанной в интегродифберенциальном виде

$$
u_{x x}+u u_{x}=\exp \left[-\frac{1}{2} \int u d x\right]\left(\sum_{i=1}^{n-1} B_{i} D_{x}^{-i} \exp \left[\frac{1}{2} \int u d x\right]\right)
$$

$n$-й элемент может быть приведен $\kappa$ линеаризованному виду $W_{(n+1) x}=B_{n-2}+$ $B_{n-1} W$, әде $W=D_{x}^{-(n-1)} \exp \left[\int u d x / 2\right]$.

ЗАмЕчАниЕ. Указанная процедура линеаризации уравнения (3.29) является естественным обобщением преобразования Коула-Хопфа, которое можно также вывести посредством $x$-обобщенного преобразования годографа для эволюционных уравнений [7].

ПРЕДЛОЖЕНИЕ 4. Для дифференииальной последователъности Бюргерса (3.16), m.e. $\partial л я$

$$
R^{n-1}[u]\left(u_{x x}+u u_{x}\right)=0, \quad n=1,2, \ldots, m,
$$

где оператор $R[u]$ задан в (3.15), $n$-й элемент может быть приведен к линеаризованному виду $v_{(n+1)}=\Omega_{n}^{n+1} v$, где $u=2 v_{x} / v u \Omega_{n}$ - произвольные постоянные.

ЗАмЕЧАниЕ. В последнем утверждении мы использовали соотношение между элементами дифференциальной последовательности Бюргерса (3.16) и последовательности из примера 2.

\section{4. ОБСУЖДЕНИЕ}

Хотя для исследования интегрируемости собственных дифференциальных последовательностей не было приведено никакой общей теоремы, данная работа предлагает определения этих объектов и подсказывает некоторые направления исследований, проиллюстрированные несколькими примерами. В частности, приведенные примеры серьезным образом указывают на то, что дополнительная последовательность и предложение 2 , которое оперирует понятиями совместности и полной совместности последовательностей могут дать полезные пути исследований интегралов собственных дифференциальных последовательностей. В этом отношении данная работа представляет интерес как начало исследований собственных дифференциальных последовательностей, но не как завершающая статья на этот предмет.

С целью получить представление о свойствах собственных дифференциальных последовательностях мы сознательно сконцентрировались на простых примерах собственных дифференциальных последовательностях, для которых общее решение 
можно вывести путем линеаризации уравнений. Напомним, что уравнения последовательности в последнем примере (последовательности Бюргерса из примера 5) линеаризуются преобразованием типа преобразования Коула-Хопфа, тогда как все другие последовательности являются примерами уравнений, линеаризуемых точечными преобразованиями. Последовательности, линеаризуемые точечными преобразованиями, имеют прекрасные свойства, связанные со структурой симметрий Ли последовательностей и с использованием дополнительной последовательности для построения полного набора первых интегралов собственных дифференциальных последовательностей. В случае последовательности Бюргерса приходится вводить нелокальные переменные для общего решения операторного уравнения $R[u] Q_{1}=0$, которое после этого приводит к дополнительной последовательности высшего порядка в терминах локальных переменных. Этот пример ясно показывает, что необходимы дальнейшие исследования подобных случаев, а именно случаев, когда в дело вступают нелокальные переменные. Мы подозреваем, что важную роль в этом исследовании сыграют нелокальные симметрии и нелокальные интегрирующие множители.

\section{ПРИЛОЖЕНИЕ}

Последовательности, рассмотренные в примерах 2-4, являются интегрируемыми, а их дополнительные последовательности сохраняют максимальную симметрийную алгебру Ли точечных симметрий, задаваемых соответствующими затравочными уравнениями. Цель данного приложения - подробное рассмотрение точечных симметрий Ли.

Найдем точечные симметрии Ли общих уравнений в системах

$$
\begin{aligned}
& u_{x x}+u_{x}^{2}=0 \\
& u_{x x}+u_{x}^{2}=e^{-u} D_{x}^{-(j-1)} c_{1}, \quad j=1,2, \ldots, m ; \\
& u_{x x}+h(u) u_{x}^{2}=0 \\
& u_{x x}+h(u) u_{x}^{2}=\exp \left[-\int h(u) d u\right] D_{x}^{-(j-1)} c_{1}, \quad j=1,2, \ldots, m,
\end{aligned}
$$

и

$$
\begin{aligned}
& u_{x x}+\lambda u_{x}-\frac{h^{\prime}(u)}{h(u)} u_{x}^{2}+h(u)=0, \\
& u_{x x}+\lambda u_{x}-\frac{h^{\prime}(u)}{h(u)} u_{x}^{2}+h(u)=h(u) D_{x}^{(j-1)} c_{1}, \quad j=1,2, \ldots, m .
\end{aligned}
$$

Очевидно, что система (П.1) есть частный случай (П.2); уравнение (П.2б) принадлежит к классу уравнений вида

$$
u_{x x}+h(u) u_{x}^{2}=\exp \left[-\int h(u) d u\right] f(x),
$$


а уравнение (П.зб) - к классу уравнений вида

$$
u_{x x}+\lambda u_{x}-\frac{h^{\prime}(u)}{h(u)} u_{x}^{2}+h(u)=h(u) f(x)
$$

где $f(x)$ берется по крайней мере из $C^{1}$. Это более чем адекватно покрывает случай многочленов в исходных уравнениях.

Если в уравнении (П.4) сделать замену зависимой переменной

$$
w=\int \exp \left[\int h(u) d u\right] d u
$$

то оно примет вид

$$
w_{x x}=f(x) .
$$

Аналогично, замена зависимой переменной

$$
w=\int \frac{d u}{h(u)}
$$

превращает уравнение (П.5) в уравнение

$$
w_{x x}+\lambda w_{x}=f(x)-1
$$

Поскольку преобразование зависимой переменной в обоих случаях является точечным, точечные симметрии Ли уравнений (П.7) и (П.9), которые несложно вычислить, приводят непосредственно к точечным симметриям Ли уравнений (П.4) и (П.5). Далее, (П.7) и (П.9) суть линейные обыкновенные дифференциальные уравнения второго порядка, поэтому каждое из них обладает восемью точечными симметриями Ли с алгеброй $s l(3, \mathbb{R})$.

Коэффициентные функции для точечной симметрии Ли уравнения (П.7),

$$
\Gamma=\xi(x, w) \partial_{x}+\eta(x, w) \partial_{w}
$$

имеют вид

$$
\begin{aligned}
\xi & =a(x)+b(x) w \\
\eta & =b_{x}(x) w^{2}+c(x) w+d(x),
\end{aligned}
$$

где функции $a(x), b(x), c(x), d(x)$ удовлетворяют уравнениям

$$
b_{x x}=0, \quad c_{x x}=b f_{x}, \quad a_{x x}=b+2 c_{x}, \quad d_{x x}=a f_{x}+\left(2 a_{x}-c\right) f .
$$


Решая эти уравнения по очереди, получаем

$$
\begin{aligned}
& b=B_{0}+B_{1} x, \quad c=C_{0}+C_{1} x+\iint\left(b f_{x}\right) d x d x \\
& a=A_{0}+A_{1} x+\iint\left(b+2 c_{x}\right) d x d x \\
& d=D_{0}+D_{1} x+\iint\left[a f_{x}+\left(2 a_{x}-c\right) f\right] d x d x .
\end{aligned}
$$

Мы не привели явный вид интегралов для произвольной функции $f(x)$, поскольку этот вид малоинформативен. Важно отметить, что интегралы имеют восемь точечных симметрий Ли.

Точечные симметрии Ли уравнения (П.9) имеют такую же зависимость от $w$, что и в (П.11a). Функции $a(x), b(x), c(x), d(x)$ должны теперь удовлетворять уравнениям

$$
\begin{aligned}
b_{x x}-\lambda b_{x} & =0, & c_{x x}+\lambda c_{x} & =b f_{x}+2 \lambda b(f-1), \\
a_{x x}-\lambda a_{x} & =2 c_{x}-3 b(f-1), & d_{x x}+\lambda d_{x} & =a f_{x}+\left(2 a_{x}-c\right)(f-1),
\end{aligned}
$$

которые легко решить; в результате мы снова имеем восемь произвольных постоянных, а потому и восемь точечных симметрий Ли.

Явные точечные симметрии Ли исходных уравнений (П.2б) и (П.3б) требуют как обращения преобразований (П.6) и (П.8), так и конкретизации функции $f(x)$. Для поддержания должного уровня простоты рассмотрим уравнение (П.1б), в котором $u=\ln w$ и $f(x)=c_{3}+c_{2} x+c_{1} x^{2} / 2$, т.е. выпишем точечные симметрии Ли уравнения, которое является полностью совместным с четвертым элементом дифференциальной последовательности (2.12):

$$
\begin{aligned}
\Gamma_{1}= & e^{-u} \partial_{u}, \quad \Gamma_{2}=x e^{-u} \partial_{u}, \quad \Gamma_{3}=6 \partial_{x}+\left(3 c_{2} x^{2}+c_{1} x^{3}\right) e^{-u} \partial_{u}, \\
\Gamma_{4}= & {\left[24-\left(12 c_{3} x^{2}+4 c_{2} x^{3}+c_{1} x^{4}\right) e^{-u}\right] \partial_{u}, } \\
\Gamma_{5}= & 6 x \partial_{x}+\left(6 c_{3} x^{2}+3 c_{2} x^{3}+c_{1} x^{4}\right) e^{-u} \partial_{u}, \\
\Gamma_{6}= & 24 x^{2} \partial_{x}+\left[24 x+\left(12 c_{3} x^{3}+8 c_{2} x^{4}+3 c_{1} x^{5}\right) e^{-u}\right] \partial_{u}, \\
\Gamma_{7}= & \left(144 e^{u}-216 c_{3} x^{2}-24 c_{2} x^{3}-6 c_{1} x^{4}\right) \partial_{x}+\left[72 c_{2} x^{2}+24 c_{1} x^{3}-\right. \\
& \left.-\left(144 c_{3}^{2} x^{3}+108 c_{3} c_{2} x^{4}+12 c_{2}^{2} x^{5}+36 c_{3} c_{1} x^{5}+7 c_{2} c_{1} x^{6}+c_{1}^{2} x^{7}\right) e^{-u}\right] \partial_{u}, \\
\Gamma_{8}= & \left(576 e^{u} x-288 c_{3} x^{3}-96 c_{2} x^{4}-24 c_{1} x^{5}\right) \partial_{x}+\left[576 e^{u}+96 c_{2} x^{3}+48 c_{1} 2 x^{4}-\right. \\
& \left.-\left(144 c_{3}^{2} x^{4}+144 c_{3} c_{2} x^{5}+32 c_{2}^{2} x^{6}+48 c_{3} c_{1} x^{6}+20 c_{2} c_{1} x^{7}+3 c_{1}^{2} x^{8}\right) e^{-u}\right] \partial_{u} .
\end{aligned}
$$

Благодарности. Настоящая работа представляет собой часть проекта, финансируемого Swedish-South African Agreement (грант № 60935), поддерживаемого со стороны sida/VR и National Research Foundation of the Republic of South Africa. П. Г. Л. Лич благодарит Department of Mathematics, Luleå University of Technology за благоприятные условия для работы в течение того времени, когда был выполнен основной объем исследований. П.Г.Л. Лич также благодарит University of KwaZulu-Natal за постоянную поддержку. 


\section{Список литературы}

[1] M. Euler, N. Euler, P. G. L. Leach, J. Nonlinear Math. Phys., 14:2 (2007), 290-310.

[2] K. Andriopoulos, P. G. L. Leach, Phys. Lett. A, 359:3 (2006), 199-203.

[3] K. Andriopoulos, P. G. L. Leach, A. Maharaj, On differential sequences, arXiv: 0704.3243.

[4] A. B. Shabat, V. V. Sokolov, "Classification of integrable evolution equations", Mathematical Physics Reviews, Soviet Sci. Rev. Sect. C Math. Phys. Rev., 4, ed. S. P. Novikov, Harwood Academic, Chur, 1984, 221-280.

[5] R. Conte (ed.), The Painlevé Property. One Century Later, CRM Ser. Math. Phys., Springer, New York, 1999.

[6] G. W. Bluman, S. C. Anco, Symmetry and Integration Methods for Differential Equations, Appl. Math. Sci., 154, Springer, New York, 2002.

[7] M. Euler, N. Euler, N. Petersson, Stud. Appl. Math., 111:3 (2003), 315-337.

Поступила в редакцию 22.05.2008, после доработки 28.08.2008 